\title{
Nontraumatic clival cerebrospinal fluid rhinorrhea
}

\author{
Rinoliquorreia espontânea secundária à fístula de clivus \\ Luana Antunes Maranhaํ, Renata de Angeli Corredato', João Cândido Araújo' \\ Serviço de Neurocirurgia do Hospital de Clínicas - Universidade Federal do Paraná, Curitiba PR, Brazil. \\ Correspondence: Luana Antunes Maranha; Rua Desembargador Motta 2.198 / apto. 130; 80420-190 Curitiba PR - Brasil;E-mail: Luanamaranha@yahoo.com.br \\ Conflict of interest: There is no conflict of interest to declare. \\ Received 08 December 2011; Received in final form 30 December 2012; Accepted 06 January 2012
}

Cerebrospinal fluid (CSF) fistula is characterized by a leak through a communication between the subarachnoid space (SAS) and the external environment. Three main factors are necessary to be present for the production of a fistula: bone defect, meningeal tear, and pressure gradient.

Spontaneous fistulae, as opposite to the posttraumatic variant, are less common. The ones submitted to a high pressure gradient are usually secondary to tumors or hydrocephalus, whereas the low-pressure gradient type may be related to congenital defects, bone erosion, excessive aeration, or encephaloceles ${ }^{1}$.

The characteristics of unilateral leak of colorless fluid through the nostril, varying with postural changes and followed by headache, must not be overlooked. While posttraumatic leaks often heal spontaneously, around $70 \%$ of the nontraumatic variant persist, with recurrences after surgical repair ranging from 25 to $87 \%^{2}$.

A patient with an upper clivus spontaneous CSF fistula is presented, among only other four previously reported cases.

\section{CASE REPORT}

A 49 year-old woman with a history of CSF rhinorrhea since 2002, had been submitted to three different attempts to surgical correction up to 2007, always through bifrontal craniotomies, with episodes of recurrent meningitis. On admission, she had no focal neurological signs except for olfactory loss. Her high blood pressure had always been poorly controlled. Magnetic resonance imaging (MRI) revealed fluid level within the sphenoidal sinus, connected to the pre-pontine cistern, through an upper clival defect (Figure).

She was then submitted to a transnasal endoscopic approach. A bone defect was identified at the upper clivus, which was repaired through the use of nasal septal cartilage secured with a mucosal flap rotation and fibrin glue. After five days, her lumbar drain was removed. She has been followed for the last four years without recurrence.

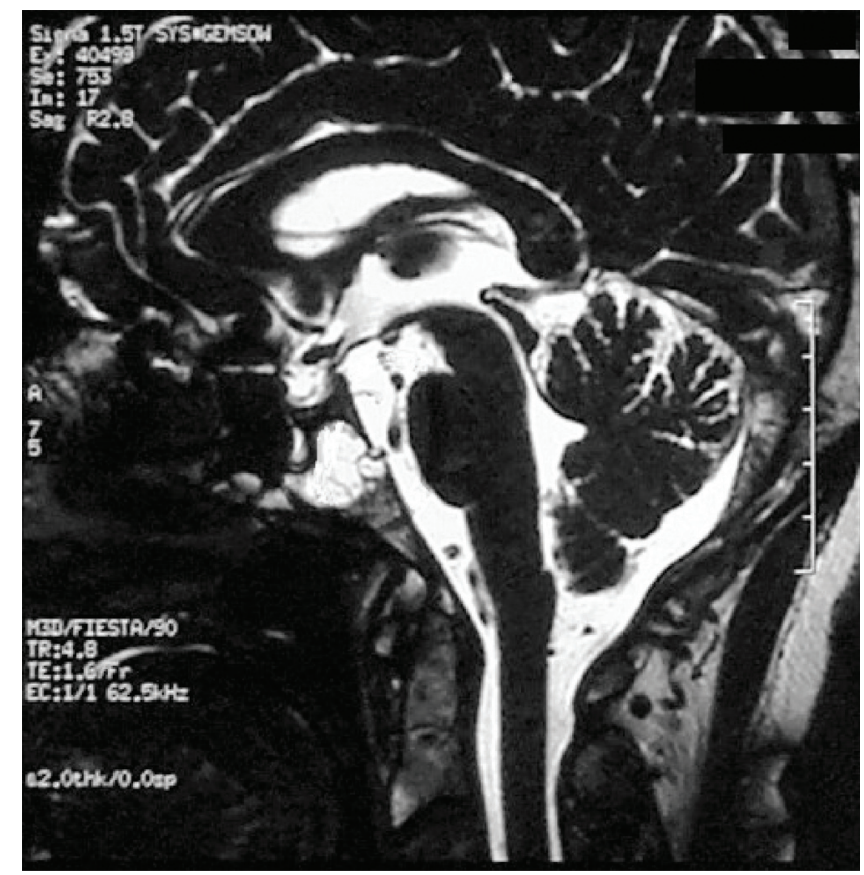

Figure. Magnetic resonance imaging T2WI shows communication between sphenoidal sinus and pre-pontine cistern

\section{DISCUSSION}

Idiopathic spontaneous CSF rhinorrhea would represent from 14 to $46 \%$ of all cases ${ }^{3}$. Skull base malformation, excessive sinus aeration, primary or secondary empty sella syndrome, and obesity would be risk factors. The association between increased weight and benign intracranial hypertension applied to a thinned skull floor has been speculated as a possible cause ${ }^{3}$. The origin of spontaneous clival fistulae remains to be elucidated.

Coitero et al. ${ }^{4}$ described the first two cases of CSF clival fistulae, mentioning the diagnostic challenges.

Aside from the primitive synchondrosis, the process of endochondral ossification for clival formation is continuous and does not allow weakened fusion points, which could eventually lead to bone defects 5 . 
Coitero et al. ${ }^{4}$ suggested that, in one of their cases, repeated basilar artery pulsations over a thinned bone structure could have produced the defect. Similar explanation could eventually be suggested for this particular case, considering in addition the associated condition of poorly controlled arterial blood pressure.
Advances in technological imaging tools have provided better facilities for the identification of CSF leaking sites, whereas improvements in surgical techniques, including the introduction of skull base endoscopy, have led to better surgical results. ${ }^{3}$. However, attempts to explain the pathophysiology of CSF clival fistulae remain speculative.

\section{References}

1. Ommaya AK. Cerebrospinal fluid rhinorrhea. Neurology 1964;14:106-113.

2. Telera S, Conte A, Cristalli G, Occhipinti E, Pompili A. Spontaneous cerebrospinal fluid rhinorrhea as the presenting symptom of sellar pathologies: three demonstrative cases. Neurosurg Rev 2007;30:78-82.

3. Elrahman HA, Malinvaud D, Bonfils NA, Daoud R, Mimoun M, Bonfils P. Endoscopic management of idiopathic spontaneous skull base fistula through the clivus. Arch Otolaryngol Head Neck Surg 2009;135:311-315.
4. Coiteiro D, Tavora L, Antunes JL. Spontaneous cerebrospinal fluid fistula through the clivus: report of two cases. Neurosurgery 1995;37:826-828.
5. Ommaya AK, DiChiro G, Baldwin M, Pennybacker JB. Nontraumatic cerebrospinal fluid rhinorrhea. J Neurol Neurosurg Psychiatry 1968;31:214-225.

\section{A patient with primary progressive aphasia developing dementia due to Alzheimer's disease}

\section{Uma paciente com afasia progressiva primária devido à doença de Alzheimer}

Thais Stvan Vaz, Paulo Henrique Ferreira Bertolucci, Fabricio Ferreira de Oliveira

Department of Neurology and Neurosurgery, Escola Paulista de Medicina, Federal University of São Paulo (UNIFESP), São Paulo SP, Brazil. Correspondence: Thais Stvan Vaz; Universidade Federal de São Paulo (UNIFESP); Escola Paulista de Medicina; Departamento de Neurologia e Neurocirurgia; Rua Botucatu 740;04023-900 São Paulo SP - Brasil;E-mail:tstvan@gmail.com

Conflict of interest: There is no conflict of interest to declare.

Received 03 January 2012; Received in final for 16 February 2012; Accepted 23 January 2012

Patients with primary progressive aphasia (PPA) often evolve to frontotemporal lobar degeneration (FTLD) syndromes ${ }^{1}$, but the logopenic form of PPA has been found to have Alzheimer's disease $(\mathrm{AD})$ pathology and in vivo biomarkers suggestive of $\mathrm{AD}^{2}$. We have reported the case of a female right-handed patient with four years of schooling, who first sought assistance at the age of 63 years-old for impairments in her speaking fluency. Her daughter mentioned that two years earlier the patient began to mumble, struggling to complete sentences, often saying things out of context, with clear perception that she meant to say something else. Semantic defects were often present, when asking for a spoon and pointing to a fork, or changing the names of her children - though she knew who they were. In the previous six months, the patient progressively lost the ability to read, currently writing with difficulty. Lately, she began to show difficulties locating herself in space. Most household tasks were effortlessly performed, but she could no longer answer the phone because she could not communicate or take notes, and neither shop, since her calculating skills had been lost.
Neurological examination was largely normal. The neuropsychiatric testing displayed the following results: a 12item neuropsychiatric inventory score of 17 (mostly for anxiety and sleep disorders), with a score of 8 for caregiver distress; Clinical Dementia Rating of 1.0; score of 9 on the Mini-Mental State Examination (orientation to time $2 / 5$; orientation to space $-3 / 5$; immediate memory $-1 / 3$; calculations $-0 / 5$; recent memory $-0 / 3$; language $-3 / 9$ ); score of 1 on the 15-point Clock Drawing Test; she was able to complete one item of the Rey-Osterrieth Complex Figure Test, but was not able to recall it; score of 3 on the Boston Naming Test (15-item short version); abstract thinking was seriously impaired, while the patient was not able to read simple sentences, and scored 2 for animals and 4 for fruits in one minute for verbal fluency tests. She was independent for activities of daily living (ADL), whereas partially independent for instrumental ADL. Magnetic resonance (MR) studies of the brain showed a pattern compatible with both PPA and AD (Fig 1 and 2). 